\title{
HIV/AIDS em região de tríplice fronteira: subsídios para reflexões sobre políticas públicas ${ }^{a}$
}

HIV / AIDS in a triple border region: subsidies for reflections on public policies VIH / SIDA en una región fronteriza triple: subvenciones para reflexiones sobre políticas públicas

Josiel Elisandro Werle ${ }^{1}$ (1) Elen Ferraz Teston ${ }^{1}$ (1) Sonia Silva Marcon² (1)

Gilmara Holanda da Cunha ${ }^{3}$ (D) Juliete Bispo dos Santos Mandu ${ }^{1}$ (B) Marcos Antônio Ferreira Junior ${ }^{1}$ (1)

1. Universidade Federal de Mato Grosso do Sul. Campo Grande, MS, Brasil.

2. Universidade Estadual de Maringá.

Maringá, PR, Brasil.

3. Universidade Federal do Ceará. Fortaleza, CE, Brasil.
Autor Correspondente:

Elen Ferraz Teston.

E-mail: elen.ferraz@ufms.br.

Recebido em 18/08/2020.

Aprovado em 19/11/2020

DOI:https://doi.org/10.1590/2177-9465-EAN-2020-0320

\section{RESUMO}

Objetivo: analisar a tendência dos casos de HIV/Aids por sexo e faixa etária nas cidades gêmeas de Mato Grosso do Sul Método: estudo ecológico de série histórica. Foram utilizadas as fichas de notificação de HIV/Aids, do período de 2009 a 2018, disponibilizadas pela Secretaria de Estado de Saúde e analisadas segundo estatística descritiva, regressão polinomial e geoespacialização dos casos. Resultados: foram registradas 734 notificações, sendo 57,9\% em homens, e com maior incidência no biênio 2017-2018 em ambos os sexos. A tendência de crescimento das notificações apresenta-se com aceleração positiva nas cidades gêmeas $(r 2=0,91 ; p<0,001)$. Os maiores coeficientes de determinação foram observados em mulheres, na faixa etária de 35 a 49 anos ( $r 2=0,98 ; p<0,001)$, e em homens de 15 a 19 anos ( $r 2=0,96 ; p<0,002)$. Conclusão: os resultados desafiam profissionais e gestores no combate à pandemia de HIV/Aids, carecendo de reflexões contínuas diante as estratégias/políticas públicas adotadas até o momento, em especial na região de fronteira. Implicações para a prática: o profissional enfermeiro, ao conhecer o perfil da sua população e a distribuição dos casos, consegue traçar estratégias capazes de atingir o público de forma eficaz e efetiva.

Palavras-chave: HIV; Síndrome de Imunodeficiência Adquirida; Saúde na Fronteira; Enfermagem; Perfil de Saúde.

\begin{abstract}
Objective: Analyze the trend of HIV/AIDS cases by sex and age in the twin cities of Mato Grosso do Sul. Method: ecologica study of historical series. The HIV/AIDS notification forms, from 2009 to 2018, made available by the Secretary of State for Health and analyzed according to descriptive statistics, polynomial regression and geospatialization of the cases were used. Results: 734 notifications were registered, $57.9 \%$ of them in men, and with a higher incidence in the biennium 2017-2018 in both sexes. The trend of growth of notifications shows a positive acceleration in twin cities $(r 2=0.91 ; p<0.001)$. The highest coefficients of determination were observed in women, in the age group 35 to 49 years ( $r 2=0.98 ; p<0.001$ ), and in men from 15 to 19 years ( $r 2=0.96 ; p<0.002$ ). Conclusion: The results challenge professionals and managers in the fight against the HIV/AIDS pandemic requiring continuous reflection on the strategies/public policies adopted so far, especially in the border region. Implications for the practice: the nurse professional, by knowing the profile of its population and the distribution of cases, is able to draw strategies capable of reaching the public effectively and efficiently.
\end{abstract}

Keywords: HIV; Acquired Immunodeficiency Syndrome; Border Health; Nursing; Health Profile.

\section{RESUMEN}

Objetivo: analizar la tendencia de los casos de VIH/SIDA por género y grupo de edad en las ciudades gemelas de Mato Grosso do Sul. Método: estudio ecológico de series históricas. Se utilizaron formularios de notificación del VIH/SIDA de 2009 a 2018, puestos a disposición por el Departamento de Salud del Estado y analizados de acuerdo con estadísticas descriptivas regresión polinómica y geospatialización de los casos. Resultados: se registraron 734 notificaciones, un $57,9 \%$ en hombres y una mayor incidencia en el bienio 2017-2018 en ambos sexos. La tendencia de crecimiento de las notificaciones se acelera positivamente en las ciudades gemelas $(\mathrm{r} 2-0,91 ; \mathrm{p}<0,001)$. Los coeficientes de determinación más altos se incluyeron en las mujeres de 35 a 49 años ( $r 2-0,98 ; p<0,001)$, y en los hombres de 15 a 19 años ( $r 2-0,96 ; p<0,002)$. Conclusión: los resultados desafían a profesionales y directivos en la lucha contra la pandemia del VIH/SIDA, carentes de reflexiones continuas frente a las estrategias/políticas públicas adoptadas hasta ahora, especialmente en la región fronteriza. Implicaciones para la práctica: el enfermero profesional, conociendo el perfil de su población y la distribución de los casos, puede diseñar estrategias capaces de llegar al público de manera efectiva y efectiva.

Palabras clave: VIH; Síndrome de Inmunodeficiencia Adquirida; Salud Fronteriza; Enfermería; Perfil de Salud. 


\section{INTRODUÇÃO}

A Síndrome da Imunodeficiência Adquirida (Aids), mesmo após três décadas de seu surgimento, ainda constitui grande desafio para a área da saúde em âmbito mundial, pois, em 2016 , ocupou o quarto lugar nas causas de morte. ${ }^{1}$ Estimativas apontam 37,9 milhões de pessoas vivendo com o vírus da imunodeficiência humana (PVHIV) no mundo, sendo que 27,3 milhões $(61,47 \%)$ possuem acesso ao tratamento antirretroviral. ${ }^{2}$

No cenário nacional, o coeficiente de mortalidade é de $5,7 / 100$ mil hab. com aumento nas regiões Norte e Nordeste e estável na região Centro Oeste. ${ }^{3}$ Ademais, a doença continua concentrada em grupos específicos, como usuários de drogas injetáveis, homens que fazem sexo com homens e profissionais do sexo, cuja prevalência é mais alta quando comparada à população geral na faixa etária de 15 a 49 anos. ${ }^{4}$

Frente a esse cenário, destaca-se a importância das políticas públicas de saúde, que a partir das demandas e necessidades evidenciadas ao longo do tempo, baseadas no perfil de saúde da população, favoreceram o desenvolvimento de leis, programas e estratégias que contribuem para o desenvolvimento de ações de cuidado. ${ }^{5}$

Atinente a isso, em 2015 o Ministério da Saúde aderiu à meta $90-90-90$, a qual propõe que $90 \%$ da população tenha conhecimento sobre seu status sorológico, $90 \%$ dos doentes esteja em tratamento e $90 \%$ dos doentes tenha carga viral indetectável. ${ }^{2}$ No entanto, alguns aspectos referentes ao sistema de saúde e educacional têm impedido o alcance da meta proposta, ${ }^{6} \mathrm{em}$ especial no que concerne à estruturação da rede de assistência às PVHIV e/ou com Aids, com enfoque no cuidado integral. ${ }^{7}$ Desse modo, embora os programas de saúde concentrem ações estratégicas comportamentais, biomédicas e estruturais, há lacunas entre a necessidade, a oferta, a adesão e retenção ao tratamento medicamentoso e não medicamentoso. ${ }^{8}$

Ademais, as características territoriais podem influenciar a distribuição, prevalência dos casos e o acesso aos serviços de saúde, em especial nas regiões de fronteira, onde se localizam as cidades gêmeas - aquelas com estreita interação política, sociocultural e econômica, apesar de localizadas em países distintos $^{9}$, o que elucida a necessidade de atenção diferenciada, sobretudo no que concerne ao acesso aos serviços de saúde. ${ }^{10}$

Salienta-se que, às vezes, a interação entre os povos ocorre devido à migração da população para território com melhores recursos. O Brasil, por exemplo, quando comparado ao Paraguai e à Bolívia, apresenta uma política pública de saúde de acesso universal, o que leva ao processo de Migração Pendular. ${ }^{11,12} \mathrm{Ou}$ seja, os indivíduos saem de sua cidade de residência/origem para outra, em busca de melhores condições, seja de acesso à saúde, trabalho ou educação, retornando no mesmo dia ou em poucos dias. ${ }^{13}$

O Sistema Nacional de Saúde boliviano, por exemplo, não é de acesso universal, as ações de cuidado são fragmentadas e os cidadãos necessitam pagar por atendimentos, sejam eles em estabelecimentos públicos ou privados. Apenas gestantes, idosos e crianças até cinco anos possuem atendimento gratuito. ${ }^{14}$ Por sua vez, no Paraguai o acesso ao sistema público de saúde é possível somente por aqueles cidadãos que possuem carteira de trabalho assinada. ${ }^{15}$

Em virtude das questões vivenciadas em regiões de fronteira, os países integrantes do Mercado Comum do Sul (MERCOSUL) realizaram acordos bilaterais para o combate e controle de agravos, influenciados pela desigualdade social, a fim de promover uma interação saudável entre as nações. Nesse sentido, o Programa de Cooperação Internacional para Ações de Prevenção e Controle do HIV/Aids estabeleceu estratégias específicas para ampliar o acesso à terapia antirretroviral em regiões de fronteira ${ }^{16,17}$, pois há maior vulnerabilidade à infecção por HIV em populações imigrantes quando comparadas à população local, seja por dificuldades de acesso aos serviços de saúde, xenofobia ${ }^{18,19}$ ou desconhecimento de direitos. ${ }^{11,20}$

Em estudo realizado na fronteira entre Brasil, Argentina e Paraguai, os autores encontraram coeficiente de incidência crescente dos casos notificados de HIV/Aids no período de 1988 a 2012, com maior prevalência da transmissão por via sexual entre heterossexuais, seguido por usuários de droga injetáveis. ${ }^{21}$ Ao considerarem as características peculiares dessa região - o intenso tráfico de drogas e o acesso livre da população estrangeira ao serviço de saúde brasileiro -, os autores asseveraram a necessidade de modificar o enfrentamento da epidemia em regiões de fronteira, ${ }^{21}$ o que justifica a realização de estudos nessa área. Cabe destacar que já existem estudos apontando as principais características da região e da população da tríplice fronteira entre Brasil, Paraguai e Argentina no estado do Paraná, ${ }^{21,22}$ mas a região de fronteira entre Brasil, Paraguai e Bolívia no estado de Mato Grosso do Sul (MS) ainda carece de estudos dessa natureza. Assim, surge o seguinte questionamento: Qual o comportamento da pandemia de HIV/Aids na região de tríplice fronteira no estado de MS?

Destaca-se que manter a vigilância contínua sobre a tendência de doenças em determinada região pode subsidiar a reorganização dos serviços oferecidos, de modo a atender as especificidades locais, mediante o desenvolvimento de ações que facilitem o acesso aos serviços de saúde e a implementação das próprias ações de cuidado.

Diante do exposto, no presente estudo tem-se o objetivo de analisar a tendência dos casos de HIV/Aids por sexo e faixa etária nas cidades gêmeas de MS.

\section{MÉTODO}

Estudo ecológico de série histórica, que analisou a tendência dos casos notificados de HIV/Aids em indivíduos a partir dos 10 anos de idade, nas cidades gêmeas localizadas no estado de Mato Grosso do Sul, no período de 2009 a 2018. O período foi determinado a fim de realizar um recorte temporal dos últimos 10 anos.

O referido estado apresenta sete cidades gêmeas: Bela Vista, Corumbá, Coronel Sapucaia, Mundo Novo, Paranhos, Porto Murtinho e Ponta Porã. Dados do último censo apontam uma população estimada de 249.521 indivíduos residentes nessas 
cidades. ${ }^{23}$ Corumbá faz divisa com Puerto Quijarro, na Bolívia e os demais municípios têm o Paraguai como país vizinho. Essas cidades apresentam uma interação denominada sinapse, o que favorece a relação entre dois ou mais países, tanto de ordem econômica quanto de livre acesso de estrangeiros a serviços oferecidos em ambos os lados. , $^{10,24,25}$

Após autorização da Secretaria do Estado e do Comitê de Ética em Pesquisa, o banco de dados, contendo as informações referentes às notificações de HIV/Aids (exceto de identificação dos indivíduos), foi enviado, de forma eletrônica, para o pesquisador principal, em dezembro de 2019. O critério de inclusão foi a notificação em uma das sete cidades-gêmeas, e aquelas que se apresentassem duplicadas seriam contabilizadas apenas uma vez.

As variáveis analisadas foram: idade, sexo, categoria de exposição, escolaridade e coeficiente de incidência bruta. Para a idade foram adotadas as seguintes faixas etárias: "10-14", "15-19", "20-34", "35-49", "50-64" e " $\geq 65$ " anos. Optou-se por essa divisão porque a faixa etária abaixo dos 19 anos apresenta-se com tendência crescente, em especial a de 15-19 anos ${ }^{26}$, o que gera a necessidade de maiores investigações. As demais faixas etárias foram agrupadas a cada 15 anos, a fim de viabilizar as análises em decorrência da distribuição do banco de dados. Os coeficientes de incidência foram obtidos a partir do número de casos de HIV/Aids notificados nas cidades gêmeas, por sexo e faixa etária, divididos pela população total, expressos por cem mil habitantes. Utilizou-se os dados do Censo Demográfico de 2010 pelo fato de as estimativas populacionais não realizarem a distribuição por sexo e faixa etária. ${ }^{23}$

Para a análise dos dados utilizou-se o software Statistical Package for the Social Sciences (SPSS) versão 23. A análise de tendência foi realizada utilizando o modelo de regressão polinomial considerando as taxas de incidência como variável dependente $(\mathrm{Y})$ e os anos como variável independente $(\mathrm{X})$, agrupados por sexo e faixa etária. A fim de evitar a colinearidade entre os termos da equação de regressão, utilizou-se a variável centralizada, sendo o ano 2013,5 o ponto médio. Diagramas de dispersão foram construídos entre a taxa de incidência e os anos, a fim de verificar a função que expressasse a relação entre eles e, com isso, escolher a ordem do polinômio para a análise, e o modelo de regressão polinomial. O coeficiente de determinação $\left(r^{2}\right)$ foi utilizado como medida de precisão. A tendência foi considerada significativa quando o modelo estimado obteve $p<0,05$.

Com o intuito de definir o modelo a ser utilizado procedeu-se à seguinte ordem dos testes: regressão linear simples $\left(Y=\beta_{0}+\beta_{1} X\right)$, e, posteriormente, os modelos de segundo grau $\left(Y=\beta_{0}+\beta_{1} X+\beta_{2} X^{2}\right)$ e de terceiro grau $\left(Y=\beta_{0}+\beta_{1} X+\beta_{2} X^{2}+\beta_{3} X^{3}\right)$. Desse modo, aquele que obteve a melhor significância estatística, maior medida de precisão e resíduos sem vícios foi o escolhido. Quando dois modelos foram semelhantes para a mesma variável, do ponto de vista estatístico, optou-se pelo mais simples, atendendo ao princípio de parcimônia. As séries foram suavizadas por meio de média móvel centrada em três médias sucessivas.
A fim de verificar a espacialidade dos casos e possíveis mudanças no local de notificação em relação ao local de moradia, procedeu-se à geoespacialização. Para tanto, utilizou-se o software QGIS, versão 2.18, para a confecção dos mapas por meio da interpolação métrica por código do município de notificação e código do município de residência de acordo com o shapefile do estado de MS disponibilizado pelo IBGE. ${ }^{27}$

O estudo foi aprovado pelo Comitê Permanente de Ética em Pesquisa com Seres Humanos da Universidade Federal de Mato Grosso do Sul, sob o Parecer 3.789.678.

\section{RESULTADOS}

No período de 2009 a 2018 foram registradas 734 notificações de HIV/Aids nas cidades gêmeas. Não houve notificações duplicadas no período. Destas, 425 (57,9\%) foram em indivíduos do sexo masculino. Do total de notificações, 37 correspondiam a indivíduos residentes no Paraguai ou Bolívia, sem identificação da cidade específica, e uma delas não tinha informação sobre o local de residência.

Em relação aos municípios onde ocorreu a notificação houve o seguinte comportamento: Ponta Porã, com 354 (48,22\%), seguido por Corumbá, com 292 (39,78\%), Mundo Novo, com $31(4,22 \%)$, Bela Vista, com 25 (3,40\%), Porto Murtinho, com 15 (2,04\%), Coronel Sapucaia, com 13 (1,77\%) e Paranhos, com quatro $(0,54 \%)$.

Observa-se o processo de migração pendular na comparação entre os mapas, Figura 1 e Figura 2, cuja transição populacional ocorre entre os municípios vizinhos, e entre os países vizinhos, ao se identificar os locais de residência. Ao longo dos anos é possível verificar o comportamento das notificações (Figura 1), sendo que no ano de 2009 foram notificados casos de Aids em Corumbá e Ponta Porã, porém, no transcorrer do tempo houve o processo de interiorização, e, em 2018, todas as cidades tiveram casos notificados.

Na Figura 2 identifica-se que o local de residência dos indivíduos notificados exibe comportamento semelhante à cronologia do local de notificação, com concentração na região Centro-Sul do estado e predomínio dos municípios de pequeno porte, exceto Ponta Porã (médio porte).

Até o ano de 2013 somente os casos de Aids eram notificados, e a incidência acumulada de 2009 a 2013 foi de 92.58 casos/100 mil habitantes. A partir de 2014, foram incluídas as PVHIV na notificação, e a incidência acumulada de 2014 a 2018 foi de 201.59 casos/100mil habitantes nas cidades gêmeas.

No período em estudo, a categoria de exposição heterossexual apresentou maior frequência, com 257 casos (35,01\%) no sexo feminino e $205(27,92 \%)$ no sexo masculino. Já a categoria de exposição homossexual acumulou 105 (14,30\%) casos. Ressaltase que em 167 (22,75\%) notificações a categoria de exposição não foi especificada, constando como ignorada.

Em relação à idade, observou-se média de 35,53 anos (mínimo 12 e máximo de 82 anos). Já referente à escolaridade, $273(37,20 \%)$ tinham, no máximo, oito anos de estudo; 203 (27,65\%), mais de oito anos; e em 258 (35,14\%) notificações essa informação foi 

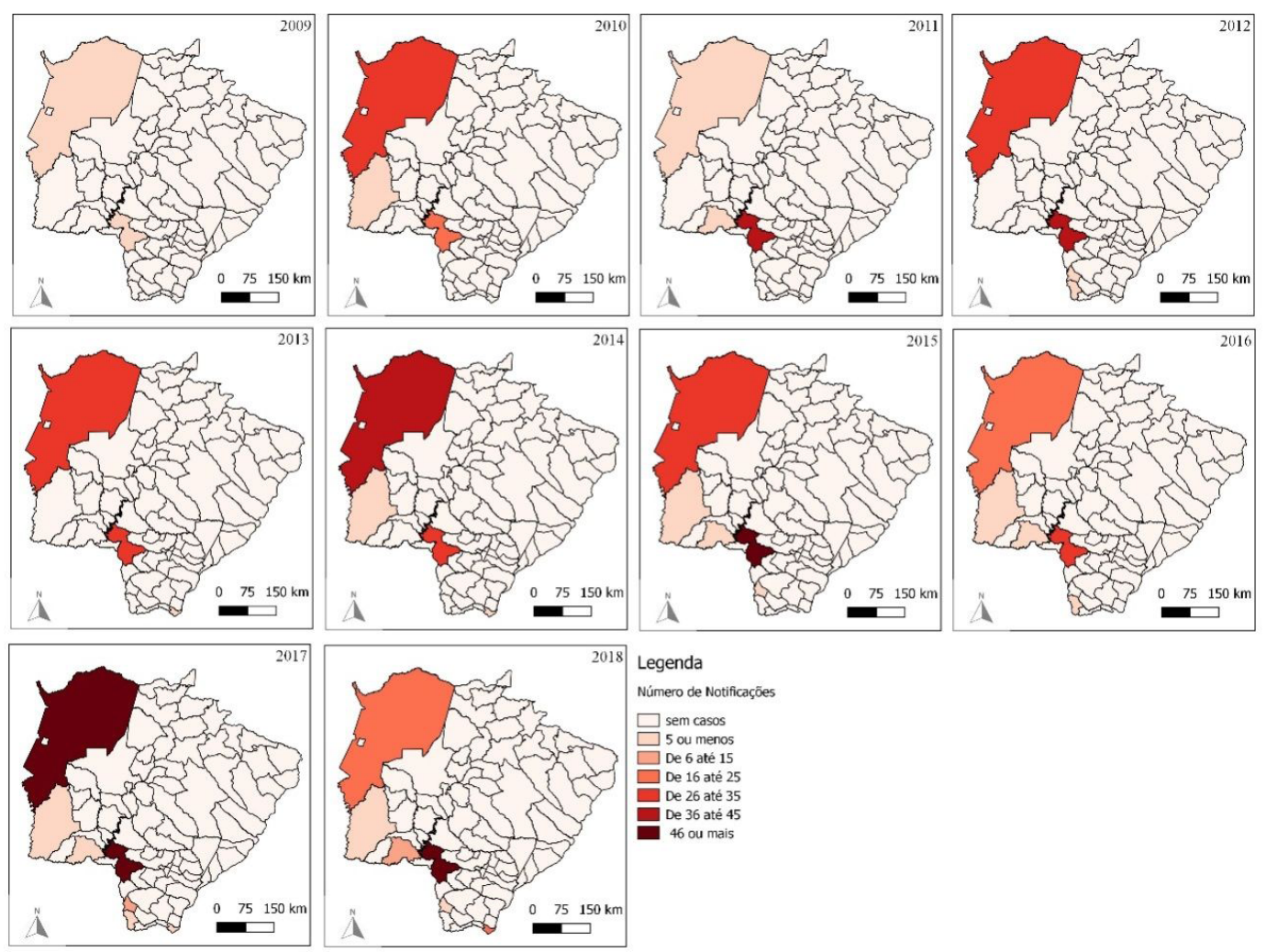

Figura 1. Total de notificações por HIV/Aids, de acordo com município de notificação por ano, Mato Grosso do Sul, 20092018. $(n=734)$.
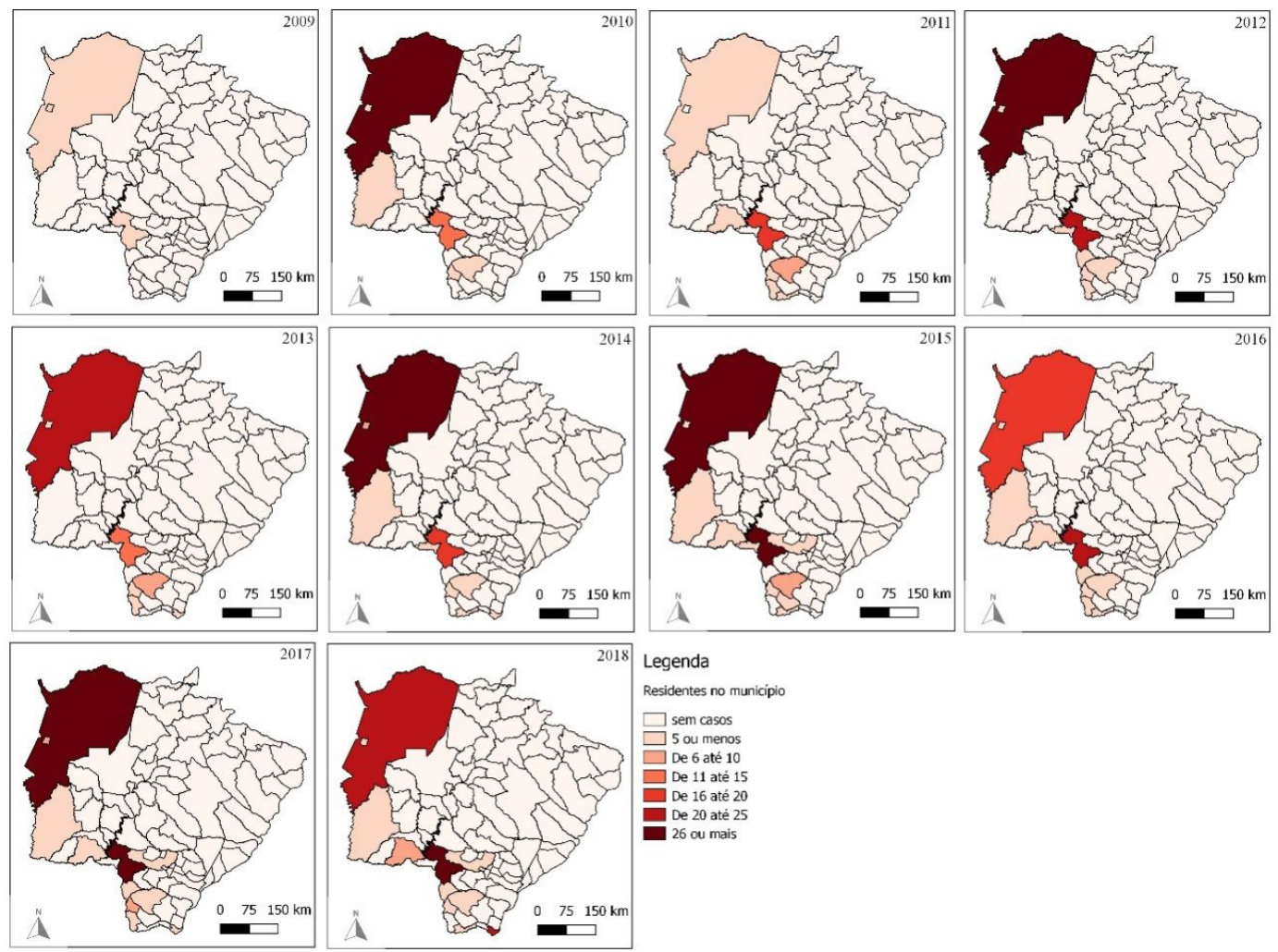

Figura 2. Total de notificações por HIV/Aids, de acordo com município de residência por ano, Mato Grosso do Sul, 2009-2018. $(n=697)$. 
ignorada. Na Tabela 1 observa-se o coeficiente de incidência distribuído por sexo e faixa etária por biênio.

$\mathrm{Na}$ análise de tendência, observou-se que os maiores coeficientes médios do período $\left(\beta_{0}\right)$ prevaleceram nos indivíduos com idade entre 20 a 64 anos para ambos os sexos, com tendência crescente e aceleração positiva, com exceção para população feminina entre 50-64 anos que se manteve estável. Evidencia-se o maior incremento anual da incidência na faixa etária de 15-19 anos para o sexo feminino $\left(\beta_{1}\right)$ e de 35-49 para o sexo masculino $\left(\beta_{3}\right)$. Não se verificou decréscimo dos casos em nenhuma faixa etária, conforme se observa na Tabela 2.

Tabela 1. Frequência absoluta e coeficiente de incidência por 100mil habitantes das notificações de HIV/Aids em cidades gêmeas, de acordo com sexo e faixa, por biênio. Mato Grosso do Sul, 2009-2018. (n=734).

\begin{tabular}{|c|c|c|c|c|c|c|c|c|c|c|}
\hline \multirow{3}{*}{$\begin{array}{l}\text { Faixa } \\
\text { etária }\end{array}$} & \multicolumn{2}{|c|}{$2009-2010$} & \multicolumn{2}{|c|}{ 2011-2012 } & \multicolumn{2}{|c|}{ 2013-2014 } & \multicolumn{2}{|c|}{ 2015-2016 } & \multicolumn{2}{|c|}{ 2017-2018 } \\
\hline & \multicolumn{10}{|c|}{ Masculino $(n=425)$} \\
\hline & $\mathbf{n}$ & inc* & $\mathbf{n}$ & inc & $\mathbf{n}$ & inc & $\mathbf{n}$ & inc & $\mathbf{n}$ & inc \\
\hline $10-14$ & - & - & 1 & 7,04 & 1 & 7,04 & - & - & - & - \\
\hline $15-19$ & - & - & - & - & - & - & 4 & 29,77 & 8 & 59,55 \\
\hline $20-34$ & 14 & 43,34 & 24 & 74,30 & 35 & 108,36 & 36 & 111,45 & 84 & 260,05 \\
\hline $35-49$ & 7 & 28,19 & 35 & 140,94 & 22 & 88,59 & 28 & 112,75 & 52 & 209,40 \\
\hline $50-64$ & 5 & 34,07 & 8 & 54,51 & 9 & 61,32 & 15 & 102,20 & 28 & 190,77 \\
\hline$>65$ & - & - & - & - & 1 & 12,41 & 3 & 37,24 & 5 & 62,07 \\
\hline Total & 26 & 24,18 & 68 & 63,25 & 68 & 63,25 & 86 & 79,99 & 177 & 164,63 \\
\hline \multirow{2}{*}{$\begin{array}{l}\text { Faixa } \\
\text { etária }\end{array}$} & \multicolumn{10}{|c|}{ Feminino $(n=309)$} \\
\hline & $\mathbf{n}$ & inc & $\mathbf{n}$ & inc & $\mathbf{n}$ & inc & $\mathbf{n}$ & inc & $\mathbf{n}$ & inc \\
\hline $10-14$ & - & - & - & - & 1 & 7,50 & - & - & 2 & 15,00 \\
\hline $15-19$ & 1 & 7,80 & 4 & 31,20 & 2 & 15,60 & 9 & 70,20 & 10 & 78,00 \\
\hline $20-34$ & 20 & 62,15 & 24 & 74,58 & 23 & 71,47 & 37 & 114,97 & 42 & 130,51 \\
\hline $35-49$ & 7 & 28,32 & 17 & 68,78 & 23 & 93,05 & 21 & 84,96 & 43 & 173,97 \\
\hline $50-64$ & 3 & 20,02 & 4 & 26,70 & 6 & 40,04 & 1 & 6,67 & 4 & 26,70 \\
\hline$>65$ & - & - & 1 & 11,48 & 2 & 22,96 & - & - & 2 & 22,96 \\
\hline Total & 31 & 29,04 & 50 & 46,84 & 57 & 53,4 & 68 & 63,7 & 103 & 96,49 \\
\hline
\end{tabular}

Fonte: Banco de dados cedido pela Secretaria do Estado de Saúde de Mato Grosso do Sul, 2009-2018 ${ }^{28} .{ }^{*}$ inc= Coeficiente incidência.

Tabela 2. Análise de tendência das taxas de notificação por HIV/Aids, de acordo com sexo e faixa etária, Mato Grosso do Sul, 2009-2018. (n=734)

\begin{tabular}{|c|c|c|c|c|c|c|c|c|}
\hline $\begin{array}{l}\text { Faixa } \\
\text { etária }\end{array}$ & Sexo & $\beta_{0}$ & $\beta_{1}$ & $\beta_{2}$ & $\beta_{3}$ & $p$-value & $\mathbf{r}^{2 *}$ & Tendência \\
\hline \multirow{2}{*}{$10-14$} & Masculino & 3,22 & $-0,39$ & $-0,65$ & & 0,07 & 0,42 & Estável \\
\hline & Feminino & 1,48 & 0,09 & 0,01 & 0,04 & 0,39 & 0,49 & Estável \\
\hline \multirow{2}{*}{$15-19$} & Masculino & 2,40 & 0,87 & 0,44 & & 0,002 & 0,96 & Crescente \\
\hline & Feminino & 20,80 & 5,01 & & & $<0,001$ & 0,86 & Crescente \\
\hline \multirow{2}{*}{$20-34$} & Masculino & 46,03 & 0,80 & 0,24 & & 0,04 & 0,58 & Crescente \\
\hline & Feminino & 44,92 & 2,94 & & & 0,005 & 0,71 & Crescente \\
\hline \multirow{2}{*}{$35-49$} & Masculino & 57,03 & $-0,60$ & 0,06 & 1,48 & 0,015 & 0,84 & Crescente \\
\hline & Feminino & 43,65 & 2,07 & 0,03 & 0,46 & $<0,001$ & 0,98 & Crescente \\
\hline \multirow{2}{*}{$50-64$} & Masculino & 42,59 & 0,91 & & & 0,002 & 0,80 & Crescente \\
\hline & Feminino & 15,98 & $-0,53$ & $-0,60$ & & 0,07 & 0,63 & Estável \\
\hline \multirow{2}{*}{$>65$} & Masculino & 9,82 & 0,96 & & & $<0,001$ & 0,92 & Crescente \\
\hline & Feminino & 6,45 & $-2,59$ & $-0,13$ & 0,27 & 0,33 & 0,53 & Estável \\
\hline Total & Ambos & 3,95 & 0,96 & & & $<0,001$ & 0,91 & Crescente \\
\hline
\end{tabular}

Fonte: Banco de dados cedido pela Secretaria do Estado de Saúde de Mato Grosso do Sul, 2009-201828* $r^{2}=$ Coeficiente de determinação. $p$-value $=0,05$ 


\section{DISCUSSÃO}

Neste estudo, observou-se tendência total crescente, com aceleração positiva dos casos de HIV/Aids na região de fronteira, no decorrer dos anos, havendo dispersão entre os municípios, o que elucida a necessidade de reflexões sobre as ações de vigilância desenvolvidas e a inclusão de particularidades de cada contexto específico, por exemplo em relação à concentração do sexo e faixa etária. No entanto, reitera-se que a mudança no critério de notificação, a partir de 2014 pode ter influenciado nestes resultados, uma vez que houve aumento na incidência acumulada de casos.

A heterogeneidade dos serviços de saúde disponíveis na América do Sul contribui para as disparidades da assistência prestada que, às vezes, se apresenta de forma segregada obrigando alguns indivíduos a buscarem assistência em outras localidades. Nos casos da Bolívia e Paraguai, a oferta do sistema de saúde caminha para o cuidado integral e universal. Já no Brasil, o Sistema Único de Saúde oferece assistência gratuita a qualquer cidadão, embora apresente características assistenciais diferentes em cada região. ${ }^{29}$

A existência do processo migratório pode levar a dificuldades para identificar as características da população, e também a disseminação de diferentes agravos à saúde. Um estudo realizado na região de fronteira da China, no período de 2010 a 2014, identificou maior prevalência de Infecções Sexualmente Transmissíveis (IST) em profissionais do sexo imigrantes do país vizinho, o Vietnã. Apontou ainda que fatores presentes na região - tráfico de drogas, exploração sexual, mão de obra barata e barreiras de acesso aos serviços de saúde - podem estar associados à prevalência das IST. ${ }^{30}$

Essa realidade não é distante do contexto de Mato Grosso do Sul, em que a região de fronteira é marcada por intenso conflito decorrente do tráfico de drogas, turismo sexual, e dificuldades econômicas dos municípios. Em um estudo realizado nessa mesma região, os autores identificaram outras doenças negligenciadas ligadas às baixas condições socioeconômicas e às barreiras de acesso aos serviços de saúde, por exemplo a Hanseníase, além do grande fluxo de pessoas em busca dos serviços de saúde no território brasileiro. ${ }^{31}$ Salienta-se que o acordo de cooperação bilateral ${ }^{17}$ possibilita o acesso de indivíduos de países vizinhos ao sistema de saúde brasileiro. Nesse contexto, os profissionais que assistem essa população necessitam reconhecer essa demanda e suas especificidades, a fim de buscarem estratégias de educação em saúde, visando à redução dos casos de novas infecções.

No entanto, algumas barreiras podem ocorrer, entre as quais o idioma, a cultura, as condições socioeconômicas e a forma de acesso do imigrante ao sistema de saúde brasileiro, mediante a utilização de meios ilegais - por exemplo o uso de endereço falso - ou procura tardia do serviço de saúde com agravamento do quadro de saúde. ${ }^{32}$ Frente a isso, o profissional de saúde, em especial o enfermeiro, que, às vezes, exerce a liderança frente à equipe multiprofissional, precisa refletir constantemente sobre as características do contexto no qual está inserido, a fim de planejar ações de cuidado que atendam as especificidades dos diferentes territórios e indivíduos.

Na comparação entre os mapas observou-se o deslocamento dos indivíduos, inclusive dentro do território nacional, pois a notificação nem sempre ocorreu no município de residência. Esse processo de migração pode estar associado a dificuldades pessoais e de infraestrutura do sistema de saúde. ${ }^{19}$ Ressalta-se que ambos os aspectos podem influenciar direta e indiretamente a vinculação com o serviço e a adesão ao tratamento. Portanto, a procura de assistência em outros municípios não deve ser coibida, mas é importante que ela seja conhecida pelos gestores, porque isso interfere no planejamento da assistência, na vigilância em saúde e nas ações a serem desenvolvidas. ${ }^{11}$

Cabe destacar que os municípios de pequeno porte ainda apresentam uma visão estigmatizada em relação à infecção por HIV. Um estudo desenvolvido no interior da Paraíba, com indivíduos provenientes da zona rural, cujo objetivo foi apreender as representações sociais em relação à Aids, apontou falas de cunho discriminatório e negativas, e relatos de não uso de preservativo pela maioria (474/789) dos entrevistados. ${ }^{33} \mathrm{~A}$ maneira de enfrentar esse tipo de situação deve ocorrer ainda na infância/ adolescência por meio das ações de educação em saúde.

Ao observar a faixa etária nas notificações destacam-se os casos de diagnóstico em jovens de 10 a 14 anos, que embora apresentem tendência estável em ambos os sexos, demonstram a iniciação sexual precoce. Em nível nacional, na mesma faixa etária, houve o registro de 211 notificações no período de 2007-2018 . ${ }^{26}$ Essa ocorrência também foi observada na região de fronteira entre Brasil, Paraguai e Argentina, em que a iniciação sexual ocorreu antes dos 14 anos de idade, principalmente em indivíduos do sexo masculino. Ressalta-se que, neste estudo, os jovens referiram não utilizar preservativo em todas as relações sexuais, mesmo tendo recebido orientações sobre sua importância. ${ }^{34}$

O fato acima mencionado merece a atenção dos estudiosos desse tema, pois esse comportamento pode ter reflexos importantes na saúde presente e futura desses jovens. É importante encontrar estratégias que alcancem esses jovens, que por vezes não respondem da mesma forma que os adultos. Nessa direção, estudo realizado na África, por exemplo, identificou baixa adesão ao uso do preservativo pela população migrante, porém, constatou que aqueles que participaram de ações educativas sobre a prática sexual segura, aumentaram em cinco vezes a chance de utilização. ${ }^{18}$

Nesse contexto, as ações de prevenção de Infecções Sexualmente Transmissíveis (IST) desenvolvidas pelos profissionais de saúde, em parceria com a escolas, por meio de diferentes abordagens, constituem fator de proteção para o início precoce de atividade sexual não segura, ${ }^{35}$ demonstrando a necessidade de fortalecer as ações intersetoriais (saúde - educação) visando à promoção de práticas sexuais seguras. ${ }^{36}$

Essas abordagens incluem a utilização de métodos alternativos de acesso e conscientização da população, seja por meio do uso de mídias sociais ou de recursos audiovisuais, 
a identificação daqueles com maior vulnerabilidade e oferta das medidas de prevenção combinada, com o intuito de o indivíduo aderir àquela que melhor encaixe em seu contexto de vida. No caso da população jovem, há necessidade de ações de estímulo à busca de testes para detecção precoce, pois muitos acreditam ser improvável possuir a infecção por HIV. ${ }^{37}$ Ademais, o comportamento da população nessa faixa etária, além de aumentar os riscos para IST, também aumenta as chances de ocorrência de outros problemas de saúde pública - a gravidez indesejada e o aborto induzido de modo não seguro. ${ }^{38}$

Por sua vez, a população com faixa etária de 15 a 19 anos também merece destaque, pois a tendência temporal de infecção por HIV/Aids, nesta região, apresentou-se crescente para ambos os sexos. No cenário nacional, para a população dessa faixa etária, no período de 2007-2018, houve 8.813 notificações, com significativa redução entre 2017 e 2018 , variando de 1.724 para 653 notificações. ${ }^{26}$ Cabe salientar que ações de vigilância contínua dos casos nas regiões de fronteira podem contribuir para o acesso ao diagnóstico e, em especial, ao tratamento, o que influencia diretamente a manutenção da carga viral indetectável e o impacto positivo na qualidade de vida. ${ }^{39}$

Em relação ao comportamento das notificações, os resultados do presente estudo corroboram a pesquisa também realizada em região de fronteira, a qual apontou o predomínio dos casos de HIV em heterossexuais. ${ }^{14}$ Ademais, o maior contingente dos casos está entre indivíduos do sexo masculino (homo e heterossexuais), o que pode estar associado à existência de múltiplas parcerias sexuais e a não utilização do preservativo, além da ausência e/ou deficiência na busca pelo serviço de saúde. ${ }^{20}$ Assim, o maior incremento anual observado na faixa etária de 35 a 49 anos em homens destaca particularidades na busca ativa tanto de ações de prevenção quanto de detecção precoce.

A tendência crescente e os maiores coeficientes médios na faixa etária de 20 a 64 anos reiteram o HIV/Aids como um problema de saúde pública. A identificação de um perfil com tendência crescente entre as mulheres demonstra a feminilização do HIV, no entanto, esse aumento pode estar associado a maior procura pelos serviços de saúde, e também a maior ocorrência de gestação na faixa etária de 20 a 40 anos..$^{40}$

Vale considerar também que a população adulta e idosa, às vezes apresenta maior prevalência de comorbidades crônicas não relacionadas à infecção por HIV/Aids, o que leva a maiores taxas de mortalidade precoce. ${ }^{41}$ Nesse sentido, destaca-se a importância de uma caracterização adicional da epidemiologia das doenças crônicas entre a população adulta e idosa que vive com HIV/Aids, a fim de otimizar o atendimento clinico e planejar estratégias eficazes de rastreamento.

Salienta-se que a modificação desse cenário vai além da atuação direta dos profissionais de saúde e perpassa os níveis da gestão municipal, estadual e federal. Os acordos de cooperação bilateral podem constituir melhores maneiras de conciliar $\mathrm{o}$ atendimento para a população de países diferentes..$^{42}$ Os profissionais, por meio da realização de atividades de educação em saúde desenvolvidas em parceria com entidades e representações locais, podem ampliar as estratégias de cuidado e acesso aos serviços.

Em relação às limitações deste estudo, destaca-se a incompletude no preenchimento de alguns dados da ficha de notificação, como local de residência, escolaridade e categoria de exposição. No entanto, ressalta-se a importância dos resultados encontrados, pois os mesmos podem proporcionar reflexões a partir das políticas públicas existentes e direcionar as ações de cuidado que atendam as especificidades das regiões de fronteira. Cabe destacar a necessidade de estudos futuros visando elucidar o perfil das notificações no estado e possibilitar comparações com regiões de fronteira.

\section{CONCLUSÃO E IMPLICAÇÕES PARA A PRÁTICA}

A tendência total dos casos de HIV/Aids, por sexo e faixa etária, em região de tríplice fronteira foi crescente. Os resultados desafiam os profissionais da saúde e gestores no combate à pandemia de HIV/Aids, carecendo de reflexões contínuas diante das estratégias/políticas públicas adotadas até o momento, em especial em região de fronteira.

Destaca-se a necessidade de ocupação dos diferentes espaços sociais pelos profissionais de enfermagem, visando à divulgação de levantamentos epidemiológicos como este, a fim de subsidiar ações e estratégias de enfrentamento.

Dessa forma, espera-se que os resultados possam nortear formas de atuação em grupos específicos, e estimular novos estudos que abordem o comportamento dos indivíduos na região de fronteira, o acesso ao serviço de saúde pela população estrangeira, as perspectivas dos profissionais de saúde da região e os impactos na saúde brasileira.

\section{FINANCIAMENTO}

O presente trabalho foi realizado com apoio da Universidade Federal de Mato Grosso do Sul - UFMS e da Coordenação de Aperfeiçoamento de Pessoal de Nível Superior - Brasil (CAPES) - Código de Financiamento 001. Bolsa de mestrado concedida a Josiel Elisandro Werle.

\section{CONTRIBUIÇÕES DOS AUTORES}

Desenho do estudo. Josiel Elisandro Werle. Elen Ferraz Teston. Coleta ou produção dos dados. Josiel Elisandro Werle. Elen Ferraz Teston

Análise de dados. Josiel Elisandro Werle. Elen Ferraz Teston. Sonia Silva Marcon. Gilmara Holanda da Cunha- Juliete Bispo dos Santos Mandu. Marcos Antonio Ferreira Junior

Interpretação dos resultados. Josiel Elisandro Werle. Elen Ferraz Teston. Sonia Silva Marcon. Gilmara Holanda da Cunha Juliete Bispo dos Santos Mandu. Marcos Antonio Ferreira Junior

Redação e revisão crítica do manuscrito. Josiel Elisandro Werle. Elen Ferraz Teston. Sonia Silva Marcon. Gilmara Holanda 
da Cunha Juliete Bispo dos Santos Mandu. Marcos Antonio Ferreira Junior

Aprovação da versão final do artigo. Josiel Elisandro Werle. Elen Ferraz Teston. Sonia Silva Marcon. Gilmara Holanda da Cunha Juliete Bispo dos Santos Mandu. Marcos Antonio Ferreira Junior

Responsabilidade por todos os aspectos do conteúdo e a integridade do artigo publicado. Josiel Elisandro Werle. Elen Ferraz Teston. Sonia Silva Marcon. Gilmara Holanda da Cunha Juliete Bispo dos Santos Mandu. Marcos Antonio Ferreira Junior

\section{EDITOR ASSOCIADO}

Gerson Luiz Marinho

\section{REFERÊNCIAS}

1. Alipour Z, Eskandari N, Mokhah S. Evaluation of knowledge and attitude of non-medical students about AIDS. J Holist Nurs Midwifery. [Internet]. 2016 [citado 2019 jun 22];26(79):10-20. Disponível em: https://www. sid.ir/en/journal/ViewPaper.aspx?ID=510635

2. Programa Conjunto das Nações Unidas sobre HIV/Aids. [Internet]. 90-90-90: uma meta ambiciosa de tratamento para contribuir para o fim da epidemia de aids. Genebra: UNAIDS; 2015 [citado 2019 jun 22]. 36 p. Disponível em: https://unaids.org.br/wp-content/uploads/2015/11/2015_11_20_ UNAIDS_TRATAMENTO_META_PT_v4_GB.pdf

3. Guimarães MDC, Carneiro M, Abreu DMX, França EB. Mortalidade por HIV/Aids no Brasil, 2000-2015: motivos para preocupação? Rev Bras Epidemiol. 2017 maio;20(20, Suppl 1):182-90. http://dx.doi. org/10.1590/1980-5497201700050015. PMid:28658382.

4. Pereira GFM, Shimizu HE, Bermudez XP, Hamann EM. Epidemiologia do HIV e aids no estado do Rio Grande do Sul, 1980-2015. Epidemiol Serv saude. 2018 nov 8;27(4):e2017374. http://dx.doi.org/10.5123/ s1679-49742018000400004.

5. Guimarães MDC, Magno L, Ceccato MDGB, Gomes RRFM, Leal AF, Knauth DR et al. HIV/AIDS knowledge among MSM in Brazil: a challenge for public policies. Rev Bras Epidemiol. 2019;22(Suppl 1):e190005. http://dx.doi.org/10.1590/1980-549720190005.supl.1.PMid:31576981.

6. Loch AP, Nemes MIB, Santos MA, Alves AM, Melchior R, Basso $\mathrm{CR}$ et al. Avaliação dos serviços ambulatoriais de assistência a pessoas vivendo com HIV no sistema único de saúde: estudo comparativo 2007/2010. Cad Saude Publica. 2018;34(2):e00047217. http://dx.doi. org/10.1590/0102-311x00047217. PMid:29489944.

7. Melo EA, Maksud I, Agostini R. Cuidado, HIV/Aids e atenção primária no Brasil: desafio para a atenção no Sistema Único de Saúde? Rev Panam Salud Publica. 2018;42:e151. http://dx.doi.org/10.26633/ RPSP.2018.151. PMid:31093179.

8. Kharsany ABM, Karim QA. HIV infection and AIDS in sub-saharan africa: current status, challenges and opportunities. Open AIDS J. 2016;10:34-48. http://dx.doi.org/10.2174/1874613601610010034. PMid:27347270.

9. Brito AR, Missio FJ. Planejamento territorial em cidades gêmeas. Rev. Bra. G\&DR. [Internet]. 2019; [citado 2020 out 24];15(6):52-63. Disponível em: https://rbgdr.net/revista/index.php/rbgdr/article/view/5184/842

10. Campos HA. The strategic role of twin cities in controlling goods in border regions in the MERCOSUR context: Uruguaiana (BR) and Paso de los Libres (AR). Redes (Bernal). 2016 dez 31;22(1):56-73. http:// dx.doi.org/10.17058/redes.v22i1.8667.

11. Rodrigues-Júnior AL, Castilho EA. AIDS e doenças oportunistas transmissíveis na faixa de fronteira Brasileira. Rev Soc Bras Med Trop. 2010 set;43(5):542-7. http://dx.doi.org/10.1590/S0037-86822010000500014. PMid:21085866.

12. Aikes S, Rizzotto MLF. Integração regional em cidades gêmeas do Paraná, Brasil, no âmbito da saúde. Cad Saude Publica. 2018;34(8):e00182117. http://dx.doi.org/10.1590/0102-311x00182117. PMid:30133667.
13. Zaslavsky R, Goulart BNG, Ziegelmann PK. Cross-border healthcare and prognosis of HIV infection in the triple border Brazil-ParaguayArgentina. Cad Saude Publica. 2019;35(9):e00184918. http://dx.doi. org/10.1590/0102-311x00184918. PMid:31508700.

14. Ferreira CMPG, Mariani MAP, Braticevic SI. As múltiplas fronteiras presentes no atendimento à saúde do estrangeiro em Corumbá, Brasil. Saude Soc. 2015;24(4):1137-50. http://dx.doi.org/10.1590/S010412902015137475.

15. Fabriz LA. Sistema Integrado de Saúde nas Fronteiras entre o Brasil e o Paraguai, no Estado do Paraná: um estudo avaliativo [tese]. Ribeirão Preto: USP; 2019. http://dx.doi.org/10.11606/T.22.2019.tde-22102019201716.

16. Ribeiro AA, de Oliveira Lupatini E, dos Santos DG. International cooperation: drugs donated by the Brazilian government, 2005-2016 Rev Panam Salud Publica. 2018;42:1-8. http://dx.doi.org/10.26633/ RPSP.2018.67.

17. Fagundes HS, Nogueira VMR, Kreutz IT. Políticas de saúde no Mercosul: relevância e funcionalidade da dimensão jurídico-norativa. Argumentum. 2018 abr 28;10(1):150-65. http://dx.doi.org/10.18315/argumentum. v10i1.18745.

18. Silvestre E, Weiner R, Hutchinson P. Behavior change communication and mobile populations: The evaluation of a cross-border HIV/AIDS communication strategy amongst migrants from Swaziland. AIDS Care - Psychol Socio-Medical Asp AIDS/HIV. 2016 fev 1;28(2):214-20. http://dx.doi.org/10.1080/09540121.2015.1081668.

19. Wu F, Zhang A, Babbitt A, Ma Q, Eyal N, Pan X et al. Overcoming HIV stigma? A qualitative analysis of HIV cure research and stigma among men who have sex with men living with HIV. Arch Sex Behav. 2018 out 1;47(7):2061-9. http://dx.doi.org/10.1007/s10508-017-1062-x. PMid:29149399.

20. Poon CM, Wong NS, Kwan TH, Wong HTH, Chan KCW, Lee SS. Changes of sexual risk behaviors and sexual connections among HIV-positive men who have sex with men along their HIV care continuum. PLoS One. 2018;13(12):1-15. http://dx.doi.org/10.1371/journal.pone.0209008. PMid:30540851.

21. Mombelli MA, Barreto MS, Arruda GO, Marcon SS. Epidemia da aids em tríplice fronteira: subsídios para a atuação profissional. Rev Bras Enferm. 2015 maio/jun;68(3):429-37. http://dx.doi.org/10.1590/00347167.2015680308i. PMid:26312513.

22. Arenhart CGM, Gomes LMX, Ribeiro EM, Barbosa TLA. Programa mais médicos em município de fronteira internacional e os desafios da gestão em saúde. Trab Educ Saúde. 2019;17(3):e0021345. http:// dx.doi.org/10.1590/1981-7746-sol00213.

23. Instituto Brasileiro de Geografia e Estatística. Censo 2010 [Internet]. Brasília: IBGE; 2019 [citado 2019 ago 22]. Disponível em: https:// censo2010.ibge.gov.br/resultados.html

24. Ministério do Desenvolvimento Regional (BR). Proposta de reestruturação do programa de desenvolvimento da faixa de fronteira. Bases de um política integrada de desenvolvimento regional para faixa de fronteira [Internet]. Brasília: MDR; 2005 [citado 2019 ago 22]. 17 p. Disponíve em: https://www.mdr.gov.br/images/stories/ArquivosSNPU/Biblioteca/ publicacoes/Introduo-e-antecedentes.pdf

25. Portaria n. 213, de 19 de julho de 2016 (BR). Estabelece o conceito de "cidades-gêmeas" nacionais, os critérios adotados para essa definição e lista todas as cidadesbrasileiras por estado que se enquadram nesta condição. Diário Oficial da União [periódico de internet], Brasília (DF), 20 jul 2016: Seção 1 [citado 2019 ago 22]. Disponível em: http:// www.in.gov.br/web/guest/materia/-/asset_publisher/Kujrw0TZC2Mb/ content/id/21772550/do1-2016-07-20-portaria-n-213-de-19-de-julhode-2016-21772471

26. Ministério da Saúde (BR). Boletim epidemiológico HIV/Aids. Brasília: Ministério da Saúde; 2018.

27. Instituto Brasileiro de Geografia. Portal de mapas do IBGE [Internet]. 2019 [citado 2019 mar 10]. Disponível em: https://portaldemapas.ibge. gov.br/portal.php\#homepage.

28. Secretaria do Estado de Saúde de Mato Grosso do Sul. Fichas de Notificações de HIV/Aids do Estado de Mato Grosso do Sul 20092018. 2019. Acesso restrito. Disponibilizado em dezembro de 2019. 
Giovanella L, Almeida PF. Atenção primária integral e sistemas segmentados de saúde na América do Sul. Cad Saude Publica 2017;33(Suppl 2):e00118816. http://dx.doi.org/10.1590/0102311x00118816. PMid:28977122.

30. Zhang C, Li X, LiuY, Qiao S, ZhouY, Tang Z et al. Human immunodeficiency virus, syphilis and hepatitis $C$ virus prevalence trends among crossborder migrant Vietnamese female sex workers in Guangxi, China BMC Public Health. 2015 dez 9;15:1223. http://dx.doi.org/10.1186/ s12889-015-2561-0. PMid:27391948.

31. Ajalla ME, Andrade SM, Tamaki EM, Waissmann W, Diettrich SH, Silva BA. The context of leprosy in Brazil-Paraguay border. Cien Saude Colet. 2016 jan;21(1):225-32. http://dx.doi.org/10.1590/141381232015211.20572014. PMid:26816179.

32. Nascimento VA, Andrade SMO. As armas dos fracos: estratégias, táticas e repercussões identitárias na dinâmica do acesso à saúde na fronteira Brasil/Paraguai. Horiz Antropol. 2018;24(50):181-214. http:// dx.doi.org/10.1590/s0104-71832018000100007.

33. Furtado FM, Santos JA, Loredanna S, Araújo E, Saldanha AA, Silva J. 30 years later: social Representations about AIDS and sexual practices of rural towns residents. Rev Esc Enferm USP. 2016;50(Spec):74-80 http://dx.doi.org/10.1590/S0080-623420160000300011.PMid:27384279.

34. Palma Priotto EMT, Führ AL, Gomes LMX, De Andrade Barbosa TL. Sexual initiation and contraceptive practices among adolescents in the tri-border area between Brazil, Argentina, and Paraguay. Rev Panam Salud Publica. 2018;42:1-9. http://dx.doi.org/10.26633/RPSP.2018.16.

35. de Moraes L, da Franca C, Silva B, Valença P, Menezes V, Colares V. Iniciação sexual precoce e fatores associados: uma revisão da literatura. Psicol Saude Doencas. 2019 mar 31;20(1):59-73. http:// dx.doi.org/10.15309/19psd200105.

36. Amaral RS, Carvalho STRF, Silva RMAM, Dias RS. Soropositividade para HIV/AIDS e características sociocomportamentais em adolescentes e adultos jovens. Rev Pesq Saúde [Internet]. 2017 mai/ago; [citado 2019 ago 22];18(2):108-13. Disponível em: http://www.periodicoseletronicos. ufma.br/index.php/revistahuufma/article/view/8384/5209

37. Clarke RD, Fernandez SB, Hospital M, Morris SL, Howard M, Wagner EF et al. Getting their feet in the door: communication cues to action for HIV testing and condom use behaviors among hispanic/latinx college students. J Prim Prev. 2020. http://dx.doi.org/10.1007/s10935-02000610-3. PMid:33033906.

38. Dulli L, Field S, Masaba R, Ndiritu J. Addressing broader reproductive health needs of female sex workers through integrated family planning/ HIV prevention services: a non-randomized trial of a health-services intervention designed to improve uptake of family planning services in Kenya. PLoS One. 2019;14(7):e0219813. http://dx.doi.org/10.1371/ journal.pone.0219813. PMid:31339919.

39. Dantas CC, Dantas FC, Monteiro BAC, Leite LJ. Perfil epidemiológico dos pacientes com HIV atendidos em um centro de saúde da região litorânea do estado de Rio de Janeiro, Brasil, 2010-2011. Arq catarinenses Med [Internet]. 2017 [citado 2019 ago 22];46(2):22-33. Disponível em: http:// www.acm.org.br/acm/seer/index.php/arquivos/article/view/250/137

40. Mesenburg MA, Wehrmeister FC, Silveira MFD. Teste de HIV solicitado e espontâneo: um estudo de base populacional com mulheres de uma cidade do Sul do Brasil. Cad Saude Publica. 2017;33(10):e00074415. http://dx.doi.org/10.1590/0102-311x00074415. PMid:29091172.

41. Croxford S, Kitching A, Desai S, Kall M, Edelstein M, Skingsley A et al Mortality and causes of death in people diagnosed with HIV in the era of highly active antiretroviral therapy compared with the general population: an analysis of a national observational cohort. Lancet Public Health. 2017;2(1):e35-46. http://dx.doi.org/10.1016/S2468-2667(16)30020-2. PMid:29249478.

42. Hortelan MS, Almeida ML, Fumincelli L, Zilly A, Nihei OK, Peres AM et al. Papel do gestor de saúde pública em região de fronteira: scoping review. Acta Paul Enferm. 2019;32(2):229-36. http://dx.doi. org/10.1590/1982-0194201900031.

${ }^{a}$ Artigo extraído da dissertação de mestrado intitulada: HIV/AIDS EM MATO GROSSO DO SUL: ANÁLISE DE TENDÊNCIA, DISTRIBUIÇÃO ESPACIAL E SOBREVIDA DOS CASOS 